\title{
PATCH CONFIDENCE K-NEAREST NEIGHBORS DENOISING
}

\author{
Cesario V. Angelino, Eric Debreuve, Michel Barlaud, Fellow IEEE \\ Laboratoire I3S, Université de Nice-Sophia Antipolis/CNRS, France \\ \{angelino, debreuve, barlaud\}ais.unice.fr
}

\begin{abstract}
Recently, patch-based denoising techniques have proved to be very effective. Indeed, they account for the correlations that exist among patches of natural images.

Taking a variational approach, we show that the gradient descent for the chosen entropy-based energy leads to a solution involving the mean-shift on patches. Then, we propose a patch-based denoising process accounting for the quality of denoising of each individual patch, characterized by a confidence. The denoised patches are combined together using each patch denoising confidence to form the denoised image.

Experimental results show the better quality of denoised images w.r.t. NL means and BM3D. The proposed method has also been tested on a professional benchmark photography.
\end{abstract}

Index Terms - Denoising, image patch, confidence, entropy, mean-shift.

\section{INTRODUCTION}

Motivated by some studies on the distribution of patches forming natural images [1, 2, 3], patch-based processing methods have recently been proposed, for example, for image and video denoising [4, 5, 6]. Indeed, these studies showed that there exist correlations among patches of natural images. As a consequence, the probability is high that patches similar to a given image patch be encountered in the image itself, offering the opportunity to recover original (unaltered/noisefree) or missing information in the patch. As a matter of fact, the nonlocal means algorithm (NL-means) [4] and BM3D [6] proved to be successful in image denoising. As in $[7,5]$ we rely on a conditional entropy criterion. In this context, our contribution is twofold. (a) Establishing a direct relation between (i) the derivative of pixel entropy conditional to its neighboring pixels and (ii) the mean shift, we propose a fully patch-based denoising procedure, i.e. a denoising of patches using patches as opposed to a denoising of pixels using patches. (b) We introduce a confidence term which measures the quality of denoised of each individual patch. This patch denoising confidence is then used to combine together each denoised patch to form the denoised image.

\section{NEIGHBORHOOD CONSTRAINED DENOISING}

\subsection{Entropy-based energy}

The inverse problem of image restoration can be formulated as a minimization problem. As mentioned in Section 1, natural images exhibit correlation among the patches which compose them. This correlation should be accounted for in deriving a restoration procedure.

Let us consider the conditional entropy $h$ of patches, i.e. the uncertainty on the color of a pixel when its neighborhood is known. Let us model an image as a random field $X$. Let $T$ be the set of pixels of the image and let $C_{t}$ be a neighborhood of pixel $t \in T$. The random vector $Y(t)=\{X(u)\}_{u \in C_{t}}$ contains the set of intensities or colors of the neighbors $C_{t}$ of pixel $t$. The random vector $Z(t)=(X(t), Y(t))$ denotes the corresponding patch, i.e. the pixel color combined with those of its neighborhood. The recovered image ideally satisfies

$$
X(t)^{*}=\arg \min _{X} h(X \mid \tilde{Y}=\tilde{Y}(t))
$$

for all $t \in T$, where $\tilde{Y}$ is the observed noisy neighborhood.

We provided a proof of adequacy of an energy such as (1) for image denoising [7].

\subsection{Derivative}

Classically, we propose to use a gradient descent procedure to solve (1). As a consequence, we need to determine the derivative of the conditional entropy of the color of a pixel knowing its neighborhood.

Entropy, whether conditional or not, can be approximated by the Ahmad-Lin estimator [8]

$$
h\left(X \mid \tilde{Y}=y_{i}\right)=-\frac{1}{|T|} \sum_{t_{j} \in T} \log p\left(x_{j} \mid y_{i}\right)
$$

where the color $x_{j}$ is encountered at pixel $t_{j}, y_{i}$ is the set of (noisy) colors in the neighborhood of $t_{j}$, and

$$
p\left(s \mid y_{i}\right)=\frac{1}{\left|T_{y_{i}}\right|} \sum_{t_{m} \in T_{y_{i}}} K\left(s-x_{m}\right)
$$

is the kernel-based estimate of the underlying conditional probability density function (PDF), with $T_{y_{i}}$ the set of pixels 
which have the same neighborhood $y_{i}, K$ a symmetric kernel, and $x_{m}$ the color encountered at pixel $t_{m}$. The derivative of (2) is

$$
\frac{\partial h\left(X \mid \tilde{Y}=y_{i}\right)}{\partial x_{i}}=-\frac{1}{|T|} \frac{\nabla p\left(z_{i}\right)}{p\left(z_{i}\right)} \cdot \frac{\partial z_{i}}{\partial x_{i}}
$$

where $p(Z)$ is the joint PDF of the high dimensional random vector $Z$.

The solution to (1) can be computed by gradient descent

$$
\left\{\begin{array}{l}
X^{(0)}=X_{0} \\
x_{i}^{(n+1)}=x_{i}^{(n)}+\beta \frac{\nabla p\left(z_{i}^{(n)}\right)}{p\left(z_{i}^{(n)}\right)} \cdot \frac{\partial z_{i}^{(n)}}{\partial x_{i}}
\end{array}\right.
$$

\section{CONFIDENCE-AWARE DENOISING}

\subsection{Patch-based denoising}

Unlike denoising methods such as [5, 4] which actually implement pixel-based iterative procedures relying on patches, as done by (5), we propose to convert (5) into a patch-based iterative procedure. Discarding the projection term $\partial z_{i}^{(n)} / \partial x_{i}$, we get

$$
z_{i}^{(n+1)}=z_{i}^{(n)}+\beta \frac{\nabla p\left(z_{i}^{(n)}\right)}{p\left(z_{i}^{(n)}\right)} .
$$

In practice, we have noticed that performing only one iteration is sufficient. The normalized derivative in (6) can be approximated by a mean shift $[9,10]$ on the high dimensional joint PDF of $Z$. In the k-th nearest neighbor $(\mathrm{kNN})$ framework, it can be expressed as [7]

$$
\frac{\nabla p\left(z_{i}\right)}{p\left(z_{i}\right)}=\frac{d+2}{\rho_{k}^{2}\left(z_{i}\right)} \frac{1}{k} \sum_{z_{j} \in k N N\left(z_{i}\right)}\left(z_{j}-z_{i}\right)
$$

where $d$ is the dimension of $Z, \operatorname{kNN}(z)$ denotes the set of the $k$ closest patches of $z$ and $\rho_{k}\left(z_{i}\right)$ is the kNN patch distance.

By setting $\beta=\rho_{k}^{2}\left(z_{i}\right) /(d+2)$ and using (7), it is clear that one iteration of the procedure (6) is equivalent to

$$
z_{i}^{*}=\frac{1}{\sum_{z_{j} \in \mathrm{kNN}\left(z_{i}\right)} w_{j}} \sum_{z_{j} \in \mathrm{kNN}\left(z_{i}\right)} w_{j} z_{j}
$$

where the weights $w_{j}$ account for the fact that, among the patches of $\mathrm{kNN}\left(z_{i}\right)$, patches farther away might not correspond as well to noisy versions of $z_{i}$ as do closer patches (they may also be slightly structurally different).

Similarly to NL-means [4],

$$
w_{j}=\exp \left(-\left|z_{i}-z_{j}\right|^{2} / \sigma_{w}^{2}\right)
$$

$\sigma_{w}$ being a parameter. To reduce the effect of noise, the distances between patches are computed after a Principal Component Analysis (PCA): $\left|z_{i}-z_{j}\right| \rightarrow\left|\operatorname{PCA}\left(z_{i}\right)-\operatorname{PCA}\left(z_{j}\right)\right|$.

\subsection{Confidence-based patch combination}

Denoised patches obtained in (8) overlap each other. In consequence, there is some redundancy in the denoising process. Indeed, for a given pixel $x_{i}$, we obtain several estimators, one for each patch to which $x_{i}$ belongs (if the patch size is $N_{p} \times N_{p}$, we have $N_{p}^{2}$ estimators.).

Clearly, among all the patches containing $x_{i}$, some will lead to an accurate denoising at $x_{i}$, some might not while leading to an accurate denoising in other pixels.

Since the noise is assumed to be uncorrelated, the synchronous summation (8) reduces the amount of noise (as characterized by its variance) by a factor of $1 / c_{i}$, where

$$
c_{i}=\frac{\left(\sum_{z_{j} \in \mathrm{kNN}\left(z_{i}\right)} w_{j}\right)^{2}}{\sum_{z_{j} \in \mathrm{kNN}\left(z_{i}\right)} w_{j}^{2}} .
$$

We call $c_{i}$ the patch denoising confidence.

In summary, for each patch $z_{i}$ of the noisy image, we are able to compute with the confidence $c_{i}$ (see (10)) a denoised patch $z_{i}^{*}$ (see (8)). These patches are then combined (or aggregated) according to their confidence term. Among a plethora of methods for combining estimators, we used, as in [6], a linear combination of denoised patches.

The denoised patches $z_{i}^{*}$ are then aggregated as follows: starting from an aggregation image of zeros and a confidence map of zeros, a denoised patch $z_{i}^{*}$ is added, after weighting by $c_{i}$, to the aggregation image at its original location, and a constant patch equal to $c_{i}$ is added to the confidence map at the same location. After dealing with all the patches, the denoised image is defined as the pointwise division between the aggregation image and the confidence map.

For a given pixel $i$, the aggregation writes

$$
x_{i}=\frac{1}{\sum_{q=1}^{N_{p}^{2}} c_{i, q}} \sum_{q=1}^{N_{p}^{2}} c_{i, q} x_{i, q}^{*},
$$

where $c_{i, q}$ is the confidence of patch $q$ among the $N_{p}^{2}$ patches which contain pixel $i$.

In Figure 1 a block diagram of the proposed PCkNN algorithm is sketched.

\section{EXPERIMENTS}

\subsection{Synthetic noisy images}

We tested our method on several images and compared to BM3D and NL means algorithms. Each image was corrupted with four levels of AWGN noise. We set BM3D in normal profile with standard parameters provided by authors. We chose for both PCkNN and NL means a patch radius of 7 and a search radius of 15 . Furthermore, as suggested from authors, we set $h=\sigma, h$ being the filtering parameter of NL means and $\sigma$ the noise standard deviation. 


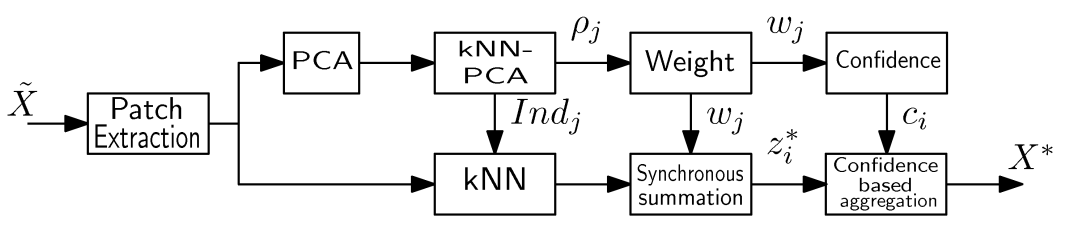

Fig. 1. Block diagram for PCkNN algorithm.
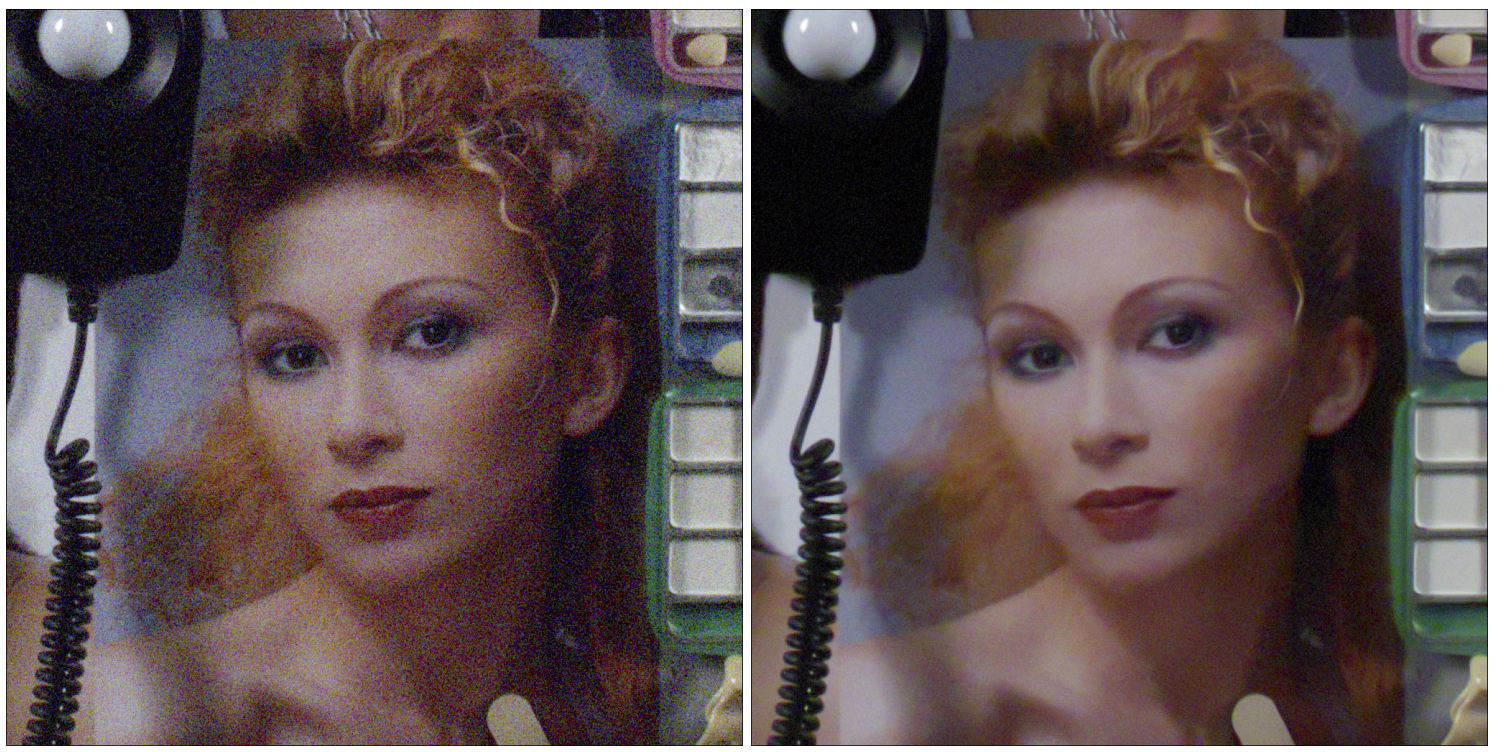

Fig. 4. Professional benchmark image (Courtesy of DxO Labs). RAW image (left) and denoised image (right).

Figure 2 shows results on Elaine image for $\sigma=25$. PCkNN outperforms NL-means and is very close to BM3D in terms of PSNR. However, the image quality of PCkNN is definitely better than the other algorithms. In particular, the residual noise is quite natural and does not exhibit spurious patterns, thus leading to a denoised image with a very natural appearance. NL-means is clearly oversmoothed and BM3D presents many flattened regions in smoothly varying areas (see Fig. 3), giving a somewhat unnatural, cartoon effect to the denoised image. This is the major drawback of this algorithm for high noise levels that might be due to the thresholding in the wavelet domain. To illustrate the cartoon effect, Figure 3 shows a close-up on the image Elaine and the corresponding isolevel lines. The orientation and density of these lines provide an indication on the direction and the norm of the gray level gradient. PCkNN preserved very well the original isolevel line configuration while BM3D created a "patchwork" of flattened regions.

\subsection{Real world noisy image}

Digital camera noise is very well approximated with an independent Gaussian stochastic process. However, contrary to the classical additive white Gaussian noise model with constant variance (used in Section 4.1 for comparison purposes), the variance of the digital camera noise can be modeled as an affine function of the signal intensity $x: \sigma^{2}(x)=\gamma x+\delta$. Moreover, most digital cameras acquire images using a single image sensor overlaid with a color filter array (CFA) which produces images with a single red, blue, or green component per pixel. The process of computing the missing 2 color components at each pixel is called demosaicing. Unfortunately, demosaicing introduces correlation among neighborhood pixels and hence correlates the noise. The result is a "structured noise" which is not Gaussian and not independent anymore. Removing this noise is a harder task since algorithms usually rely on a hypothesis of independence. Thus, we applied it to each color channel of the raw image, i.e., before demosaicing. Furthermore, we adapted our denoising algorithm to the varying variance model by making $\sigma_{w}^{2}$ in (9) equal to $\gamma \bar{x}+\delta, \bar{x}$ being the average patch intensity. After denoising the image has been demosaiced. Figure 4 shows a result on a 1024x1024crop out of a 4416x3312-DxO Labs benchmark image taken with a Canon G10 at ISO 1600. Again, the denoised image has a very natural appearance without flattened regions.

\section{DISCUSSION}

This paper presented PCkNN a fully patch-based denoising algorithm. Denoised patches are provided with a confidence 


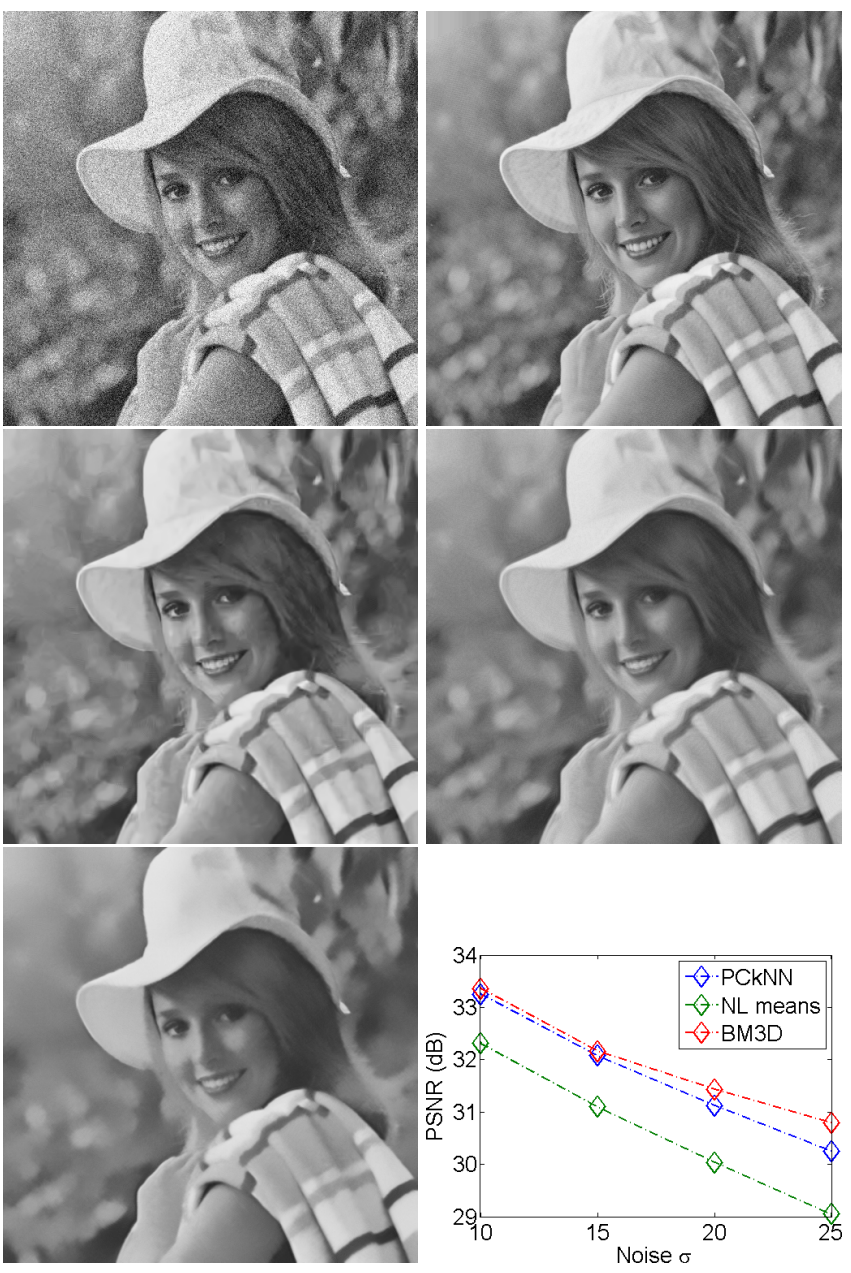

Fig. 2. In lexicographic order: Noisy, Original, BM3D, PCkNN, NL-means, and PSNR plot. The image Elaine was corrupted with an additive white Gaussian noise with standard deviation of $\sigma=25$.

term which measures the quality of denoising. These confidence terms are then combined together with (denoised) patches in order to reconstruct the denoised image. Experimental results clearly show the better quality of PCkNN denoised images w.r.t. state of the art denoising techniques. In particular, denoised images have a very natural appearance. Image details are well preserved and there is no cartoon effect even in high levels of noise. PCkNN has also been tested on a professional DxO Labs benchmark image giving very good and promising result. As mentioned in Section 4.2, digital cameras image denoising is a challenging task since noise variance is function of the signal.

\section{Acknowledgments}

The authors would like to thank DxO Labs for providing the raw test image of Figure 4 and the demosaicing software.

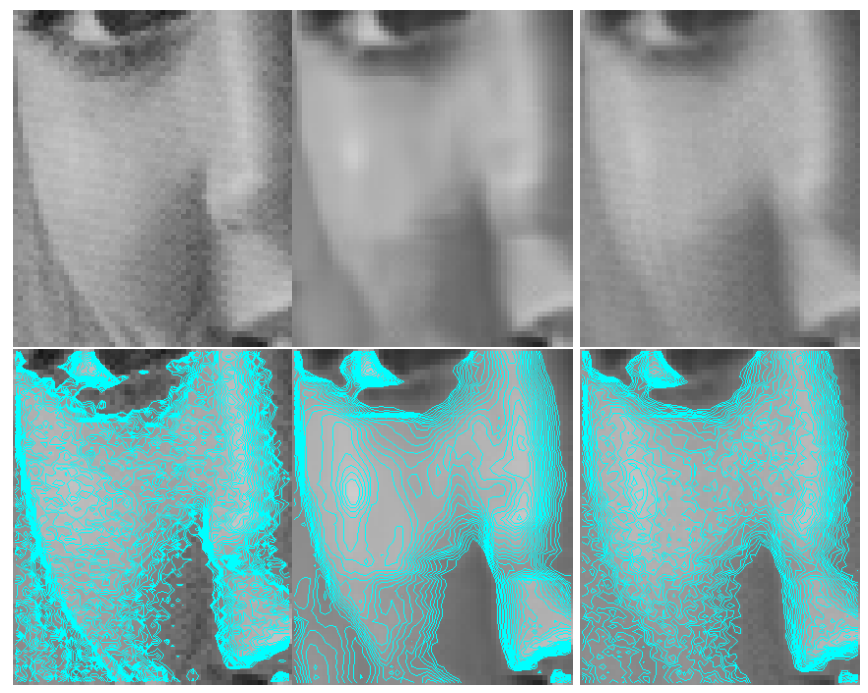

Fig. 3. A close-up on the image Elaine. From left to right: Original, BM3D, and PCkNN. First row: image alone; second row: isolevel lines superimposed on the image.

\section{REFERENCES}

[1] G. Carlsson, T. Ishkhanov, V. de Silva, and A. Zomorodian, "On the local behavior of spaces of natural images," Int. J. Comput. Vision, vol. 76, pp. 1-12, 2008.

[2] J. Huang and D. Mumford, "Statistics of natural images and models," in IEEE CVPR, 1999, pp. 541-547.

[3] A. B. Lee, K. S. Pedersen, and D. Mumford, "The nonlinear statistics of high-contrast patches in natural images," Int. J. Comput. Vision, vol. 54, no. 1-3, pp. 83-103, 2003.

[4] A. Buades, B. Coll, and J.M. Morel, "A non-local algorithm for image denoising," in IEEE CVPR, Washington, DC, USA, 2005, pp. 60-65.

[5] S. P. Awate and R. T. Whitaker, "Unsupervised, informationtheoretic, adaptive image filtering for image restoration," IEEE Trans. Pattern Anal. Mach. Intell., vol. 28, no. 3, pp. 364-376, 2006.

[6] K. Dabov, A. Foi, V. K., and K. Egiazarian, "Image denoising by sparse $3 \mathrm{~d}$ transform-domain collaborative filtering," IEEE Trans. Image Process., vol. 16, pp. 2007, 2007.

[7] C. V. Angelino, E. Debreuve, and M. Barlaud, "Image restoration using a knn-variant of the mean-shift," in IEEE ICIP, San Diego, CA, USA, October 2008.

[8] I. A. Ahmad and P. Lin, "A nonparametric estimation of the entropy for absolutely continuous distributions," IEEE Transactions On Information Theory, pp. 372-375, 1976.

[9] K. Fukunaga and L. D. Hostetler, "The estimation of the gradient of a density function, with applications in pattern recognition," IEEE Trans. On Inf. Theory, vol. 21, pp. 32-40, 1975.

[10] D. Comaniciu and P. Meer, "Mean shift: A robust approach toward feature space analysis," IEEE Trans. Pattern Anal. Mach. Intell., vol. 24, pp. 603-619, 2002. 